\title{
Evaluation of the potential for energy saving in macrocell and femtocell networks using a heuristic introducing sleep modes in base stations
}

\author{
Willem Vereecken*, Margot Deruyck, Didier Colle, Wout Joseph, Mario Pickavet, Luc Martens and Piet Demeester
}

\begin{abstract}
In mobile technologies two trends are competing. On the one hand, the mobile access network requires optimisation in energy consumption. On the other hand, data volumes and required bit rates are rapidly increasing. The latter trend requires the deployment of more dense mobile access networks as the higher bit rates are available at shorter distance from the base station. In order to improve the energy efficiency, the introduction of sleep modes is required. We derive a heuristic which allows establishing a baseline of active base station fractions in order to be able to evaluate mobile access network designs. We demonstrate that sleep modes can lead to significant improvements in energy efficiency and act as an enabler for femtocell deployments.
\end{abstract}

\section{Introduction}

Compared to the different wired access network technologies, mobile access networks are significantly less energy efficient [1]. In light of ICT being estimated to be responsible for about $2-4 \%$ of the worldwide carbon emissions [2,3], this is an important challenge and energy efficiency is a key design parameter for current and future mobile access networks.

On the other hand, in mobile communications the user bit rate demand is ever increasing. In the past, mobile access networks were mainly used for voice and text communication, However, data communications are rapidly increasing and currently responsible for the larger part of the traffic on mobile access networks [4].

The above trends pose an important challenge for the future mobile access network. On the one hand, it needs to sustain the increasing user demand, which corresponds with deploying an increasing number of base stations, while at the same time it is required to limit carbon emissions and energy consumption.

\footnotetext{
* Correspondence: willem.vereecken@intec.ugent.be Ghent University - IBBT; Department of Information Technology (INTEC), Internet Based Communication Networks and Services - Wireless \& Cable, Gaston Crommenlaan 8, bus 201, B-9050 Ghent, Belgium
}

One solution is the optimisation of the base stations in order to make them more energy efficient. Also, new network technologies like long term evolution (LTE) [5] are emerging, which will allow higher bit rates as well as higher ranges to provide these bit rates. It is however questionable whether these solutions will suffice since the rising bit rate demand implies a drastic increase in the number of base stations. Thus, solutions that reduce the power consumption of a mobile access network as a whole are required.

When optimising a system for energy efficiency, the introduction of sleep modes is one of the most commonly used approaches and is already well known in other wireless communication systems such as sensor networks [6]. In this article, we will demonstrate the introduction of sleep modes is essential if we want to deploy mobile access networks with a high coverage for large bit rates. Moreover, we will provide a method to establish the best-effort active base station fraction in such a network. We will limit our discussion to LTE implementations as this is the emerging standard designed for high bit rate applications [5]. This can be used as a baseline to evaluate the energy efficiency of practical implementations.

In Section 2, we elaborate on the work related to our study. In Section 3, we discuss the base station behaviour. 
This is used as an input for a heuristic to find an optimal distribution of active base stations, which is derived in Section 4. We apply the heuristic on a theoretical topology to evaluate the active base station fraction (Section 5) and then evaluate the environmental impact of the different design choices (Section 7). Afterwards, in Section 8 we demonstrate the use of the heuristic in a topology designed for an urban environment. In Section 9 we summarise the main conclusions.

\section{Related work}

Sleep modes in wireless networks are already used in multiple situations. For example, the requirement of providing a certain coverage while certain nodes can sleep is a well known problem in sensor networks $[7,8]$. The authors of [6] give a good overview of the different techniques that can be used in wireless contexts. Due to the requirement for long battery life times, sleep modes were also introduced in the mobile subscriber devices $[9,10]$.

In this article, we are focussing on the mobile access network and more specifically the base station. In some studies, it is suggested to reduce the carbon footprint of a base station by using renewable energy sources $[11,12]$. Although this effort is viable in itself, it doesn't improve the energy consumption of the mobile access network. Other authors make improvements on the base stations themselves [13]. These efforts are viable but, as we will demonstrate, optimisations in the network as a whole are possible as well.

In terms of introducing sleep modes in base stations, some solutions are already suggested. Some authors make the distinction between micro-sleep where base stations are only shortly suspended and deep-sleep where users need to connect to different base stations in order to maintain connectivity $[14,15]$. In this study we do not consider these micro-sleep modes. Also, in [16], it is demonstrated that sleep modes are an enabler for introducing small cell base stations and low power mobile access networks. In $[17,18]$, a similar exercises are performed to evaluate the switching off of macrocells in order to limit power consumption of the access network. In the above cited studies, the network optimization is performed without taking into account the user behaviour. However, the authors of [19] propose an algorithm in which also the requirements of the users are accounted for.

Another approach which is used is the so called cell zooming where during low use periods base stations are turned off and only lower bit rates are fully covered $[20,21]$. This technique however only allows a limited fraction of base stations to be switched off and reduces the level of service for the users since the approach does not account for user behaviour.

In the above cited studies, the focus is on the optimization of current network deployments. As such, the performance evaluation of the solution is based on an always-on network. In this study, we would like to investigate the question on the maximum number of sleeping base stations while guaranteeing the required connectivity for the users. This while taking into account the future high bit rate requirement of the users. This question is relevant as the future mobile access network will require a significant increase in energy efficiency.

Based on this result, one can establish a baseline to assess the energy efficiency of future practical implementations. We will demonstrate that for these high bit rate mobile access network, a disruptive approach with large idle base station fractions is possible and necessary. Moreover, we offer a heuristic on the basis of which algorithms and protocols for this future mobile access network can be designed.

\section{Characterisation of base stations}

When characterising the power consumption of a mobile network, one needs to consider that the power consumption of the base station is more or less constant. This is a direct consequence of the input power of the antenna, which directly defines the power consumption, being kept at a constant level $[22,23]$. On the other hand, the range of the base station varies depending on which bit rate is needed. The range and the bit rate are thus strongly correlated. When deploying a mobile network for a certain bit rate, this will have consequences for the base station density and thus for the overall power consumption of a network.

To determine the range of a base station, first the maximum allowable path loss to which a transmitted signal can be subjected while still being detectable at the receiver, is calculated [22]. Therefore, a link budget has to be set up. A link budget takes all the gains and the losses from the transmitter through the medium to the receiver into account. When the maximum allowable path loss is known, the range can be determined by using a propagation model. Different propagation models exist and the propagation model used depends on the design parameters (e.g. indoor vs. outdoor, macrocell vs. femtocell base station, urban vs. suburban vs. rural area, etc.). In this study, we focus on macrocell and femtocell base stations. For the macrocell base station we used the Erceg $C$ model [24], while for the femtocell base station the ITU-R P.1238 model is used [25]. For both the macrocell and the femtocell we assume a frequency of $2.6 \mathrm{GHz}$ as defined in the LTE standard. We assume the macrocell to transmit at 43 $\mathrm{dBm}$, whereas femtocells transmit at $21 \mathrm{dBm}$.

When determining the bit rate at which we want to evaluate the range, two parameters are key: the receiver signal-to-noise ratio (SNR) and the channel bandwidth. The receiver SNR represents the SNR at the receiver for 
a certain bit error rate (BER) and is determined by the used modulation scheme and coding rate.

The modulation scheme provides the translation of a binary bit stream into an analogue signal that can be transmitted through the medium. This is done shifting both amplitude and phase of the signal, a number of distinct modulations result in the modulation scheme. LTE supports three modulation schemes: quadrature phase shifting keying (QPSK) shifting only on phase and allowing 2 bits (4 values) per symbol and 16-QAM and 64-QAM (quadrature amplitude modulation), shifting both amplitude and phase allowing for 4 bits (16 values) and 6 bits (64 values) per symbol, respectively.

The modulation scheme is always accompanied by a coding rate that allows to determine if there are any errors introduced in the signal during transmission. This is done by adding redundant bits to the signal. The coding rate is the number of real information bits divided by the total number of bits. For example, a coding rate of $2 / 3$ implies that for every two information bits one redundant bit is added. In LTE, the following combinations are supported: 1/3 QPSK, 1/2 QPSK, 2/3 QPSK, 1/ 2 16-QAM, 2/3 16-QAM, 1/2 64-QAM, and 2/3 64QAM. The higher the coding rate and the higher the modulation, the higher the bit rate, but also the higher the receiver SNR and thus the lower the range becomes. For a macrocell base station in a $5 \mathrm{MHz}$ channel, for example, a range of $1089.9 \mathrm{~m}$ is obtained for a bit rate of $2.8 \mathrm{Mbps}(1 / 2 \mathrm{QPSK})$ and $193.5 \mathrm{~m}$ for a bit rate of 16.9 Mbps (2/3 64-QAM).

A second important parameter that influences the bit rate and the range is the channel bandwidth. The channel bandwidth indicates the width of the frequency band used to transmit the data. The higher the channel bandwidth, the higher the bit rate, but the lower the range. For a macrocell base station, the 1/2 QPSK modulation corresponds to a bit rate of $2.8 \mathrm{Mbps}$ and a range of $1089.9 \mathrm{~m}$ in a $5 \mathrm{MHz}$ channel, while it corresponds with a bit rate of $11.3 \mathrm{Mbps}$ and a range of $778.7 \mathrm{~m}$ in a 20 MHz channel.

The bit rates obtained with varying channel bandwidth, modulation scheme and coding rate are summarised in Table 1 The relation between bit rate and range that is thus obtained is displayed in Figure 1. We displayed the results for LTE femtocells and macrocells at a channel bandwidth of $5 \mathrm{MHz}$ and $20 \mathrm{MHz}$.

\section{Heuristic to put base stations in sleep mode}

\subsection{Determination of the heuristic}

In Section 3, we demonstrated that higher bit rates correspond with shorter distances between the user and the base station. Since the bit rate demand is on the rise, mobile networks will be required to provide coverage for these higher bit rates. This implies smaller cell sizes,
Table 1 Bit rates resulting from modulation schemes, coding rates and channel bandwidths in LTE

\begin{tabular}{lcc}
\hline Channel BW: & $\mathbf{5} \mathbf{~ M H z}$ & $\mathbf{2 0} \mathbf{~ M H z}$ \\
\hline 1/3 QPSK & $2.8 \mathrm{Mbps}$ & $11.3 \mathrm{Mbps}$ \\
1/2 QPSK & $4.2 \mathrm{Mbps}$ & $16.9 \mathrm{Mbps}$ \\
2/3 QPSK & $5.7 \mathrm{Mbps}$ & $22.5 \mathrm{Mbps}$ \\
1/2 16-QAM & $8.5 \mathrm{Mbps}$ & $33.8 \mathrm{Mbps}$ \\
2/3 16-QAM & $11.3 \mathrm{Mbps}$ & $45.1 \mathrm{Mbps}$ \\
1/2 64-QAM & $13.3 \mathrm{Mbps}$ & $54.1 \mathrm{Mbps}$ \\
2/3 64-QAM & $16.9 \mathrm{Mbps}$ & $67.6 \mathrm{Mbps}$ \\
\hline
\end{tabular}

resulting in a larger number of base stations to cover a certain area and hence larger power consumption. On the other hand, since cell sizes are smaller, there is also a smaller number of users present in a cell and thus the probability of a user requiring the high bit rates in a cell is decreasing. This consideration leads to the opportunity to introduce sleep modes in the network. If all users in a cell can be served by base stations outside the cell, then the base station in the cell is no longer required and can be turned to a sleep mode. Hence, the power consumption of the mobile network can be reduced.

In order to evaluate this opportunity, we designed a heuristic to find a best-effort distribution of base stations to be put to sleep mode in order to minimise power consumption.

Initially, the topology of the base stations and the distribution of the users and their associated bit rate demand are considered to be known. We assume the number of users as ' $m$ ' and the number of base stations as ' $n$ '. The vector $U$ contains the coordinates of the users. The vector $\mathbf{R}$ contains the required range of the users. It is calculated based on the bit rate requirement of the user which is then mapped on the corresponding base station range. The matrix $\mathbf{B}$ contains the coordinates of the base stations. We now construct an $m \times n$ matrix $\mathbf{P}$ :

$$
\mathbf{P}_{i j}=\left\{\begin{array}{l}
1 \text { if }\left|\mathbf{B}_{j}-\mathbf{U}_{i}\right| \leq \mathbf{R}_{i} \\
0 \text { if }\left|\mathbf{B}_{j}-\mathbf{U}_{i}\right|>\mathbf{R}_{i} .
\end{array}\right.
$$

This matrix represents the possibilities to provide a user with a suitable connection. In case a row of the matrix $\mathbf{P}$ contains only zeroes, this implies that no base station is close enough to the user to provide its demand. Since we can do nothing to serve those users we need to keep them out of the equation.

For the base stations, we assume the capacity limits to be larger than the actual capacity required by the base station. This is a valid assumption since LTE is currently designed providing sufficient resource blocks to cover the expected load. Moreover, this also implies that 


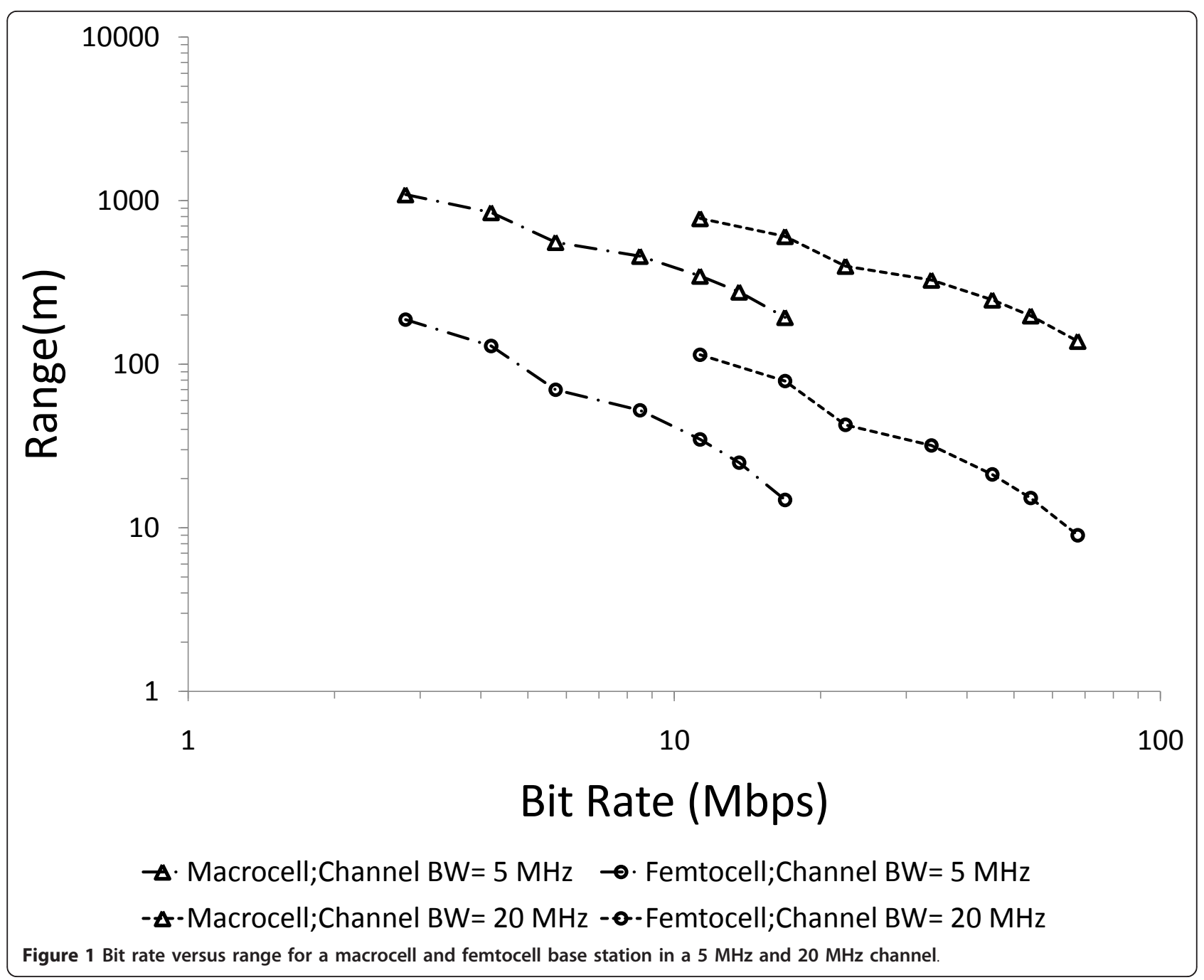

extending the capacity of base stations is also possible in case our design should require it.

Based on the matrix $\mathbf{P}$, we can now construct two vectors:

$$
\begin{aligned}
& \mathbf{C}^{\mathbf{u}}: \mathbf{C}_{i}^{\mathbf{u}}=\sum_{j=1}^{n} \mathbf{P}_{i j} \\
& \mathbf{C}^{\mathbf{b}}: \mathbf{C}_{j}^{\mathbf{b}}=\sum_{i=1}^{m} \mathbf{P}_{i j}
\end{aligned}
$$

In $\mathbf{C}^{\mathbf{u}}$ one can determine the number of base stations that can serve a certain user. On the other hand, in $\mathbf{C}^{\mathbf{b}}$ the number of users potentially connecting to a base station are expressed. Based on this information, we can now iteratively switch on base stations until all users are connected. Since in every iteration the number of active base stations increases, we aim to keep the number of iterations as low as possible. Therefore we propose the following strategy:

1. Identify the users with the least potential connections.

2. Select the base stations to which these users can connect.

3. Of these base stations, select the base station to which most users can connect and switch it on.

4. Remove the users that connect to this base station from the heuristic and start over.

By first satisfying the users with the least connections we assure that later on in the algorithm we do not need to switch on an additional base station just to satisfy this one user. On the other hand, by assuring that in each iteration the potential base station satisfying the most users is selected, in each step we maximise the number of users we no longer need to consider. Thus, 
we simplify the problem and reduce the number of iterations.

In the algorithm, we can implement this by defining an $\mathrm{n}$-dimensional vector $\mathbf{S}$. We initialise this vector on $\mathbf{S}_{j}=0$ and for each base station that is switched on, we put the value on 1 . Since we remove the users that are satisfied at each iteration, the heuristic is stopped once all users are satisfied. The algorithm is denoted in pseudocode in Algorithm 1.

Algorithm 1. Heuristic for a best-effort distribution of sleep
modes in a base station topology for minimal power
consumption
repeat
$\mathbf{C}^{\mathbf{u}}: \mathbf{C}_{i}^{\mathbf{u}}=\sum_{j=1}^{n} \mathbf{P}_{i j}$
$\mathbf{C}^{\mathbf{b}}: \mathbf{C}_{j}^{\mathbf{b}}=\sum_{i=1}^{m} \mathbf{P}_{i j}$
$\mathbf{I}=$ Find $\left(i \mid \mathbf{C}_{i}^{\mathbf{u}}=\operatorname{Min}\left(\mathbf{C}^{\mathbf{u}}\right)\right)$
$\mathbf{Z}: \mathbf{Z}_{j}=\operatorname{Sign}\left(\sum_{i \in \mathbf{I}} \mathbf{P}_{i j}\right)$
$\mathbf{C}^{\mathbf{b}}=\mathbf{C}^{\mathbf{b}} \times \mathbf{Z}^{(*)}$
$\mathbf{J}=$ Find $\left(j \mid \mathbf{C}_{j}^{\mathbf{b}}=\operatorname{Min}\left(\mathbf{C}^{\mathbf{b}}\right)\right)$
$\mathbf{S}_{\mathbf{J}_{1}}=1^{(* *)}$
$\mathbf{Q}=\mathbf{P} \circ \mathbf{S}$
for $j=1 \rightarrow n$ do
if $\mathbf{Q}_{j}=1$ then
$\mathbf{P}_{* j}=0$
end if

\section{end for}

until $\sum_{i, j} \mathbf{P}_{i j}=0$

(*) ' $x$ ' represents elementwise multiplication.

$(* *)$ We select only one base station to be switched on, hence $\mathbf{J}_{1}$.

The heuristic requires information on both the exact location of the base stations and the users, as well as the bit rate requirement of the users. In practical deployments, the user information will not be readily available. Additionally, the user information is only valid at a certain moment in time and is subject to change due to movement and changes in bit rate requirements. Reevaluation on regular intervals for an entire mobile access network could be resource and time intensive.

On the other hand, the heuristic does provide a nearoptimal solution for putting deployed base stations in a sleep mode. Hence, it provides a baseline for practical implementations of sleep mode algorithms in mobile access networks to be evaluated against. Also, during the design of a mobile access network, it can provide useful information on the suitability of a topology for the introduction of sleep modes.

\subsection{Use of the heuristic in a theoretical case}

In order to determine the potential and consequences of sleep modes, we evaluate the heuristic in a theoretical base station topology. For the base stations, we define a series of bit rates at which they can operate and the corresponding ranges (cf. Figure 2). We define a triangular grid on which we deploy the base stations. This results in a surface with hexagonal cells. The side length $r$ of the cell, which is also the largest possible distance between a user and a base station (i.e. when the user is at the geometric centre between three base stations) is defined by range corresponding to the largest bit rate for which we want to provide coverage. An example of this topology can be seen in Figure 3, which we will discuss later.

Next, we define a user density and distribute users randomly in the area covered by the base stations until the required user density is reached. We use a uniform distribution. Since users on the edge of the covered area can reach less base stations than users in the centre, we need to make sure the covered area is large enough to limit this effect.

As mentioned before, the different users will require different bit rates. In order to model this, we use a distribution $\varphi$ representing the probability a user requires a certain bit rate. We consider this distribution to be exponential:

$$
\phi(B R) \propto \frac{1}{B R^{\alpha}}
$$

In (4), $\varphi$ represents the probability of a user requiring a bit rate $B R$. The preference for lower/higher bit rates is determined by $\alpha$. For $\alpha>0$ there is a larger probability for lower bit rates, $\alpha=0$ results in a uniform distribution and $\alpha<0$ implies a preference for larger bit rates. The probability distribution is normalised so that

$$
\sum_{B R} \phi(B R)=1
$$

We have displayed this probability distribution for the different available bit rates for LTE at a channel bandwidth of $5 \mathrm{MHz}$ in Figure 2. One can see how the parameter $\alpha$ defines the distribution. A preference for lower bit rates $(\alpha>0)$ is typical in a situation where the use of the mobile access network is mainly for voice calls and the use of high bit rate applications like video streaming is limited. Also, cases in which idle users maintain a low bit rate connection with the access network, can be modelled by using a high value for $\alpha$. Situations where the larger part of the users need to maintain a high bit rate connection with the access network can be modelled with negative values for $\alpha$. 


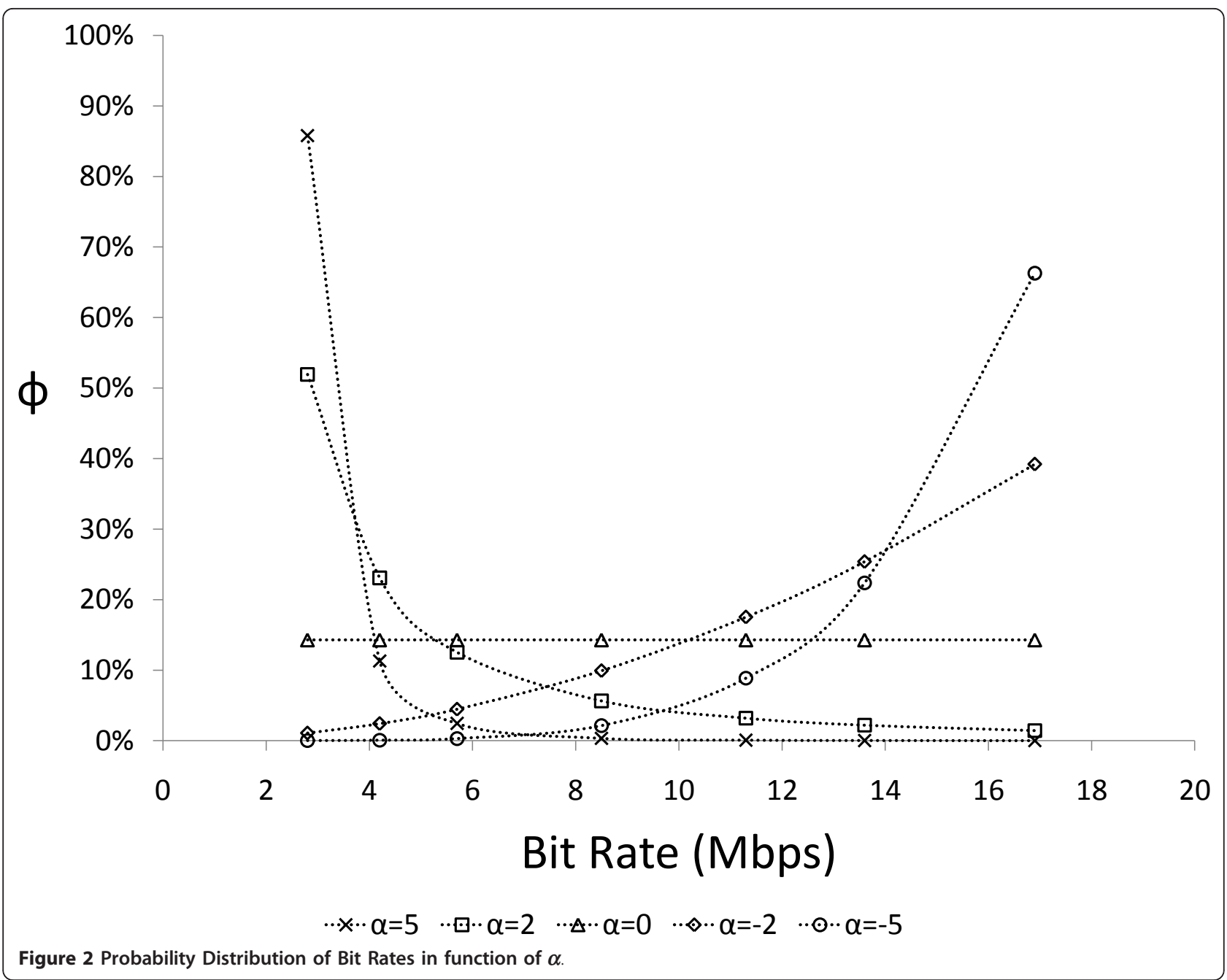

In Figure 3 we display an example distribution for a user density $D_{U}$ of 500 users $/ \mathrm{km}^{2}$ with $\alpha=3$ in a femtocell access network operating for a channel bandwidth of $5 \mathrm{MHz}$. In the access network there are 1951 base stations, covering an area of approximately $1.11 \mathrm{~km}^{2}$. Of these base stations, 42 need to be active in order to provide all users with a connection. This leads to an active base station fraction of $2.15 \%$. In the following discussion, we will denote this as $F_{A}$ expressing the number of active base stations divided by the total number of base stations. Lower values for $F_{A}$ imply more base stations are switched off and thus power consumption is reduced.

\section{The active base station fraction $\boldsymbol{F}_{\boldsymbol{A}}$}

\subsection{Upper limit for $F_{A}$}

In Figure 3 we see that, contrary to macrocell deployment [22], the density of the base stations $D_{B}$ is higher than the user density $D_{U}$. This is a direct consequence of the requirement to cover the area for a high bit rate and thus with a low range for the base stations. If we regard the special case where every user needs a dedicated base station, it is clear that because of the lower user density, not all base stations need to be switched on. As a consequence, we get an upper limit for the fraction of active base stations $F_{A}$ by requiring, the density of the active base stations is equal to the user density. This results in the following representation:

$$
F_{A}<\frac{D_{U}}{D_{B}}
$$

If $r$ is the range at the highest bit rate, the surface of a cell is:

$$
\frac{3 \sqrt{3}}{2} r^{2}
$$

Assuming there is one base station per hexaconal cell, we obtain for (6):

$$
F_{A}<D_{U} \frac{3 \sqrt{3}}{2} r^{2}
$$




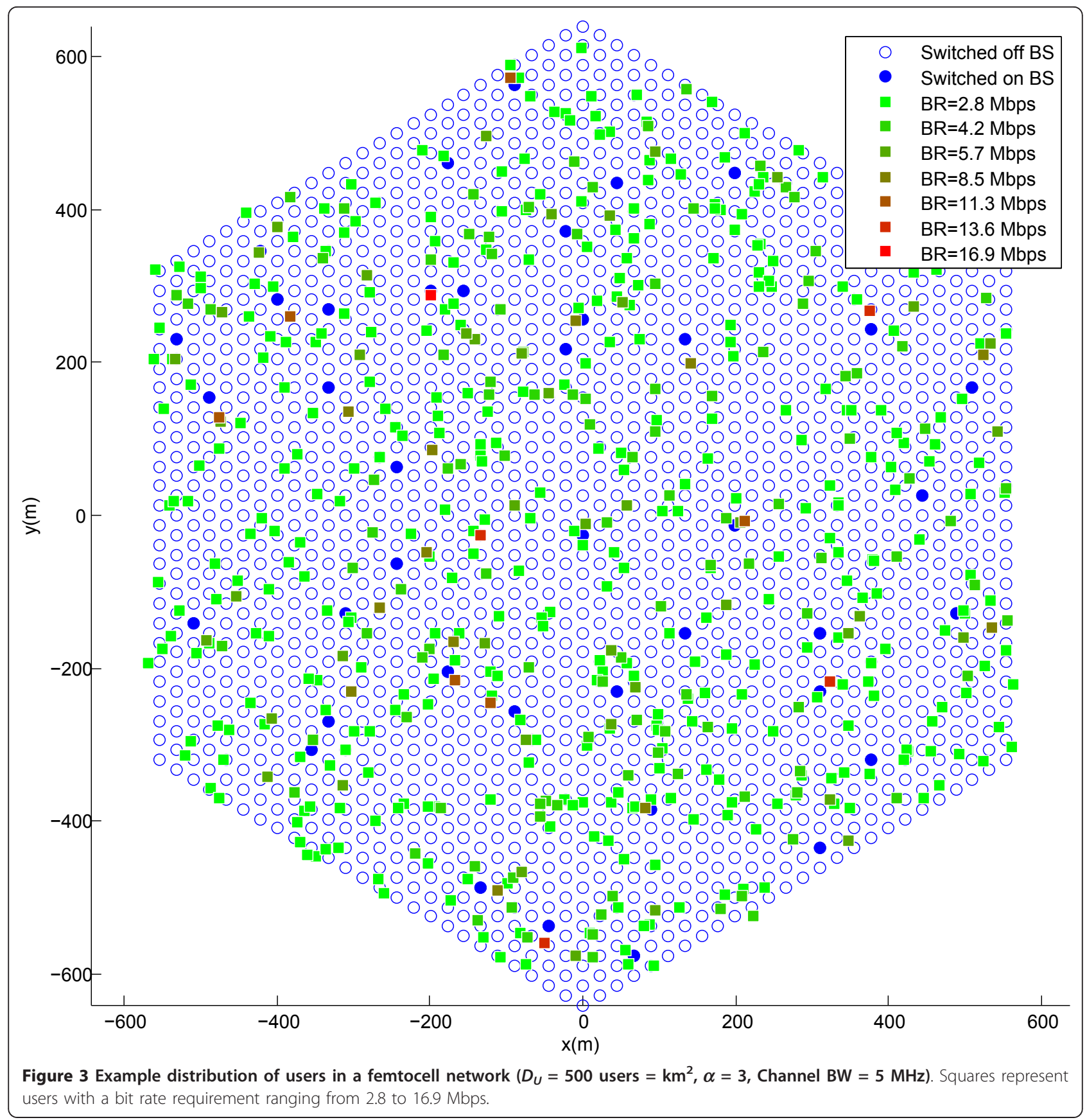

This consideration remains valid as long as the user density is smaller than the base station density:

$$
D_{U}^{\max }<\frac{1}{\frac{3 \sqrt{3}}{2} r^{2}}
$$

Using the ranges determined in Section 3, in the case of a femtocell network with a $5 \mathrm{MHz}$ channel bandwidth this corresponds with a user density of approximately 1757 users $/ \mathrm{km}^{2}$. In comparison, for a macrocell network this limit is already reached at a user density of 10 users $/ \mathrm{km}^{2}$.

\subsection{Determination of $F_{A}$ through simulation}

As one can see in Figure 3, users are not evenly distributed over the surface and some clusters appear. Additionally, the users operating at lower bit rates can connect to base stations at larger distances. Therefore, lower active base station fractions will be possible. 
In order to evaluate this, we simulated user distributions of 500 users $/ \mathrm{km}^{2}$ in a hexagonal access network of 1951 base stations with varying $\alpha$ and a channel bandwidth of $5 \mathrm{MHz}$ and $20 \mathrm{MHz}$. For each case, we performed 25 simulations of which we display the average result and the standard deviation, represented by error bars. The result is displayed in Figure 4a. We also performed the same exercise with macrocell networks. Here we used a hexagonal access network of 469 base stations. This result is displayed in Figure 4b.
When using femtocells (Figure 4a), even in cases with a large preference for high bit rates $(\alpha=-5)$ the fraction of active base stations is lower than the theoretical maximum. For high values of $\alpha$, additional savings in the order of 85 to $95 \%$ compared to the theoretical maximum are possible. Note however that for $\alpha>3$ the probability for a user requiring a high bit rate is so low that the viability of deploying such a dense network is questionable.

It is also important to note the standard deviation on the simulations. Depending on the user distribution the

(a) Femtocells

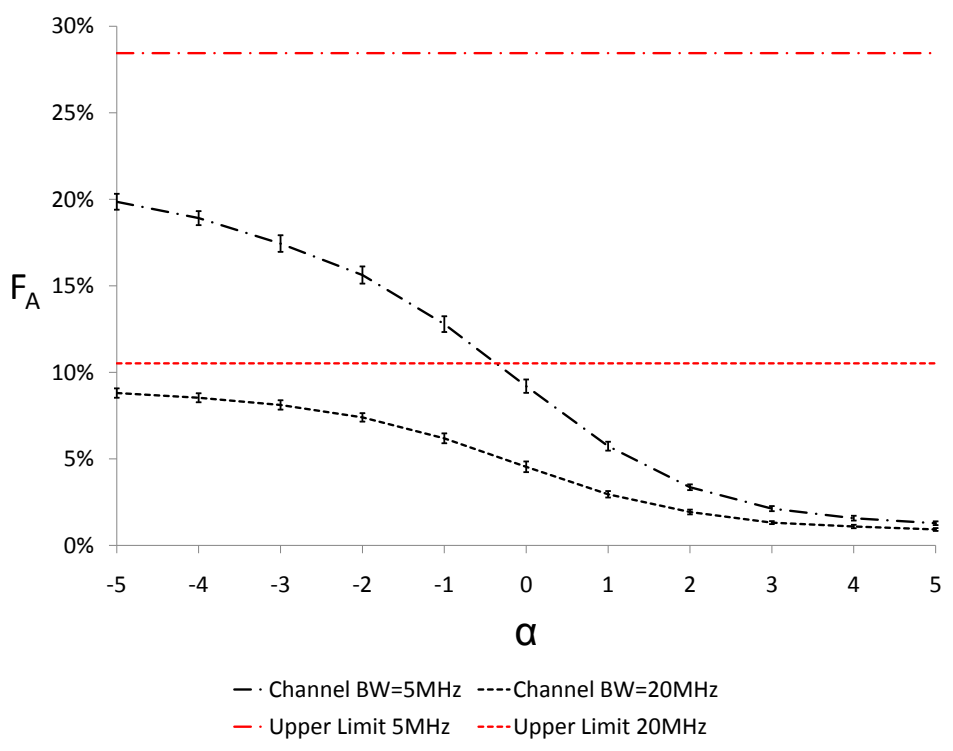

(b) Macrocells

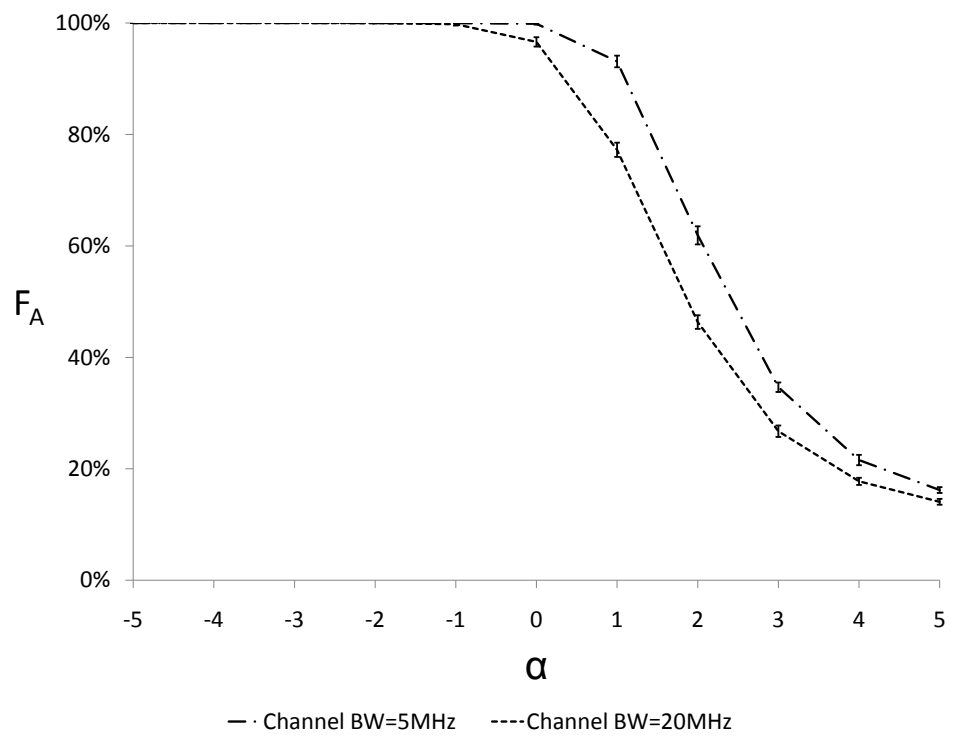

Figure 4 Influence of on the active base station fraction $F_{A}$. 
result may vary 2 to $10 \%$. This indicates that the actual fraction of active base stations is difficult to predict.

In the case we use macrocells (Figure $4 \mathrm{~b}$ ), it is also possible to save energy using sleep modes, albeit in the case where lower bit rates are preferred over higher bit rates $(\alpha>0)$. Active base station fractions of $15-25 \%$ for $\alpha=5$ can lead to significant reductions in power consumption although the active base station fractions in the order of $50-75 \%$ seem more realistic.

Finally, we evaluate the influence of the user density on the active base station fraction. Figure 5a shows the result of 100 simulations per case for varying user densities and channel bandwidths when using femtocells with $\alpha=3$. We also displayed the theoretical upper limit as described by (6). One can see that although the theoretical limit is increasing with increasing user density, the simulations result in a slower increase of active base stations. Even when using macrocells, the number of users can become significantly high before reaching an active base station fraction of $100 \%$ (Figure $5 b$ ).

\section{Evaluation of the heuristic towards optimal solutions}

In the above sections, we used derived our results based on the presented heuristic. However, it is important to evaluate the proximity of the results compared

(a) Femtocells

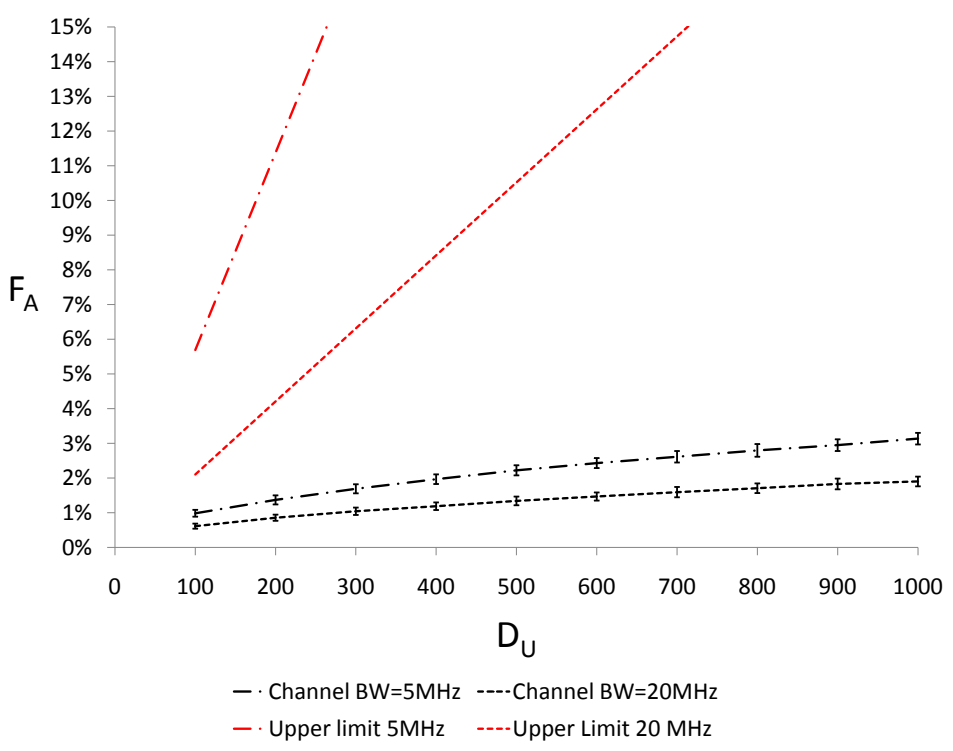

(b) Macrocells

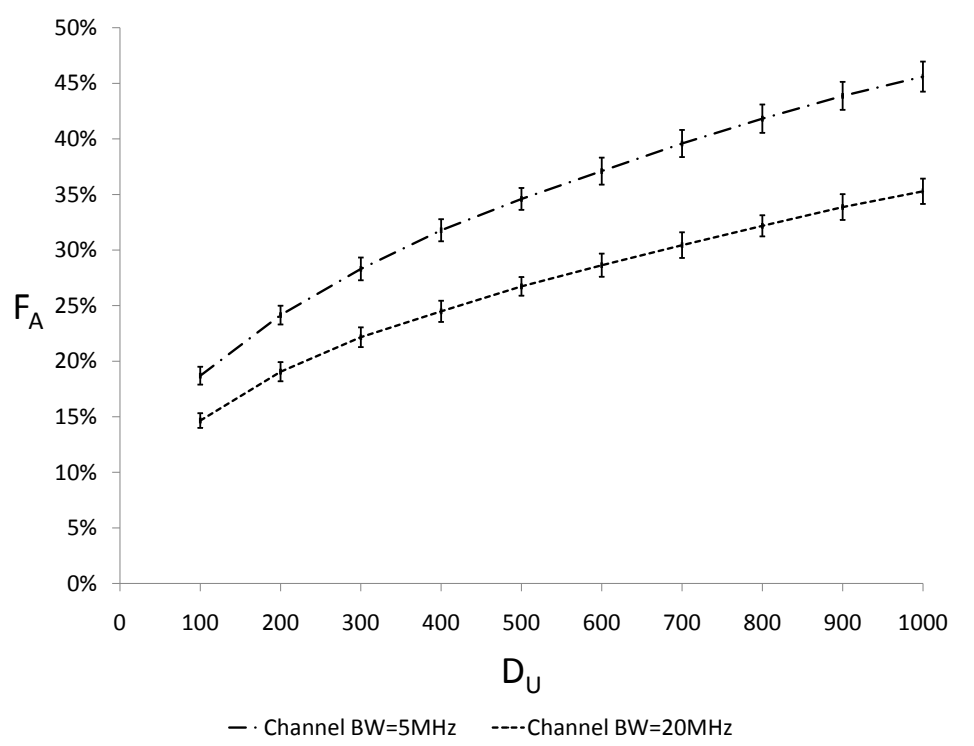

Figure 5 Influence of $D_{U}$ on the active base station fraction $F_{A}(\alpha=3)$. 
to the optimal solution. The optimal solution can be derived using integer linear programming (ILP). Using the variables introduced in Section 4.1, we define the objective function by demanding a set of active base stations in which as little base stations as possible are active.

$$
\text { Minimise : } \sum_{j=1}^{n} \mathbf{S}_{j}
$$

As functional constraint, we specify that each user needs to be able to connect to at least one base station. Note that the matrix $\mathbf{P}$ is considered as an input for the ILP.

$$
\forall i: \sum_{j=1}^{n} \mathbf{P}_{i j} \mathbf{S}_{j} \geq 1
$$

Finally, we need to inform the ILP solver that there is only two possibilities for each value of $\mathbf{S}$, either on (1) or off (0). This is denoted by the following sign constraint:

$$
\forall j: S_{j} \in(0,1)
$$

We compare the heuristic to the ILP solution in a femtocell setup with channel bandwidth of $5 \mathrm{MHz}$. The access network consists of 1951 base stations. The user density varies between 200 and 1000 users $/ \mathrm{km}^{2}$ and $\alpha$ varies between -5 and 5 . We calculated $\mathbf{P}$ and then solved the problem using both ILP and the heuristic. The ILP problem is solved with the IBM ILOG CPLEX Optimiser [26]. The difference between both is displayed in Figure 6. Each point represents a calculation originating from the same $\mathbf{P}$. Note that the same number of active base stations does not imply the same set of active base stations.

First of all, it is clear that the ILP always provides the optimal solution. Second, for higher base station densities, the heuristic results in a near-optimal solution. For lower densities, there is a significant difference between the

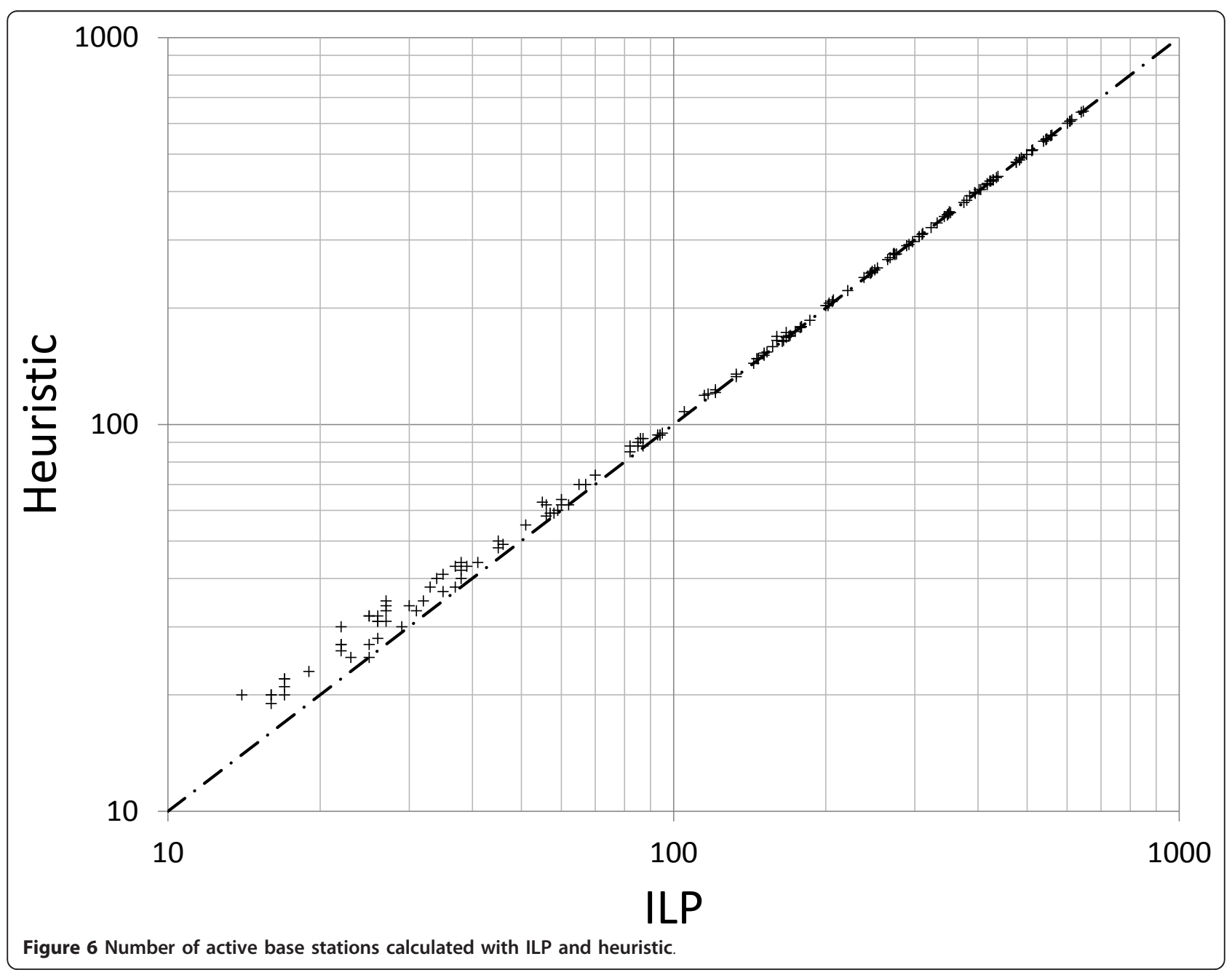


heuristic and the ILP. These values are mainly corresponding with $\alpha \geq 4$.

This deviation can be explained by the heuristic being designed to first satisfy the users requiring the highest bit rate, and particularly the bit rate defining the base station density. These users are (almost) not present for the high values of $\alpha$. As such, for every user there is a high number of possible base stations connecting each user and picking one of these base stations will immediately drastically reduce the solution space, possibly excluding the optimal solution.

Although this is a factor to be considered when using the heuristic, it is of little impact to the overall conclusions, as it only marginally impacts the conclusions for very low base station densities. Moreover, this is an extreme case in which it is doubtful whether it is economically viable to roll out an access network capable of these high bit rates when there are almost no users requiring them.

\section{Environmental impact of the mobile access network}

\subsection{Power consumption}

One of the purposes of the introduction of sleep modes is reducing the environmental footprint. However, this evaluation is largely dependent on the selected environmental parameter. First we investigate the power consumption. On the one hand we determine the power consumption when the base station is active, $P_{\text {act }}^{F}$ or $P_{\text {act }}^{M}$ for femtocell base stations or macrocell base stations, respectively. On the other hand, there is the power consumption $P_{s}^{F}$ or $P_{s}^{M}$ when the base stations are in sleep mode.

When we want to evaluate the power consumption of the access network, we need to multiply the average power consumption of a base station by the number of base stations in the network. This number can be directly derived from the base station density $D_{B}$ and the covered area, which is invariable depending on the design choices. Hence, if we want to evaluate power consumption regardless of the magnitude of the covered area, we can consider the power consumption density $D_{P}$ :

$$
D_{P}=D_{B}^{*}\left[P_{s}^{*}+F_{A}^{*}\left(P_{a c t}^{*}-P_{s}^{*}\right)\right] \quad(*=F, M)
$$

with $F_{A}^{F}$ or $F_{A}^{M}$ the average active base station fraction of the access network and $D_{B}^{F}$ or $D_{B}^{M}$ the base station density.

If we assume a typical power consumption of $10 \mathrm{~W}$ for a femtocell base station and $3 \mathrm{~kW}$ for a macrocell base station, in the case of $D_{U}=500$ users $/ \mathrm{km}^{2}, \alpha=3$, a channel bandwidth of $5 \mathrm{MHz}$ and assuming the ideal situation where $P_{s}^{*}=0$, a macrocell network would consume 10.7
$\mathrm{kW} / \mathrm{km}^{2}$, whereas a femtocell network would consume only $0.37 \mathrm{~kW} / \mathrm{km}^{2}$ or $3.5 \%$ of what the macrocell network would consume. Alternatively, when assuming the base stations cannot be put in sleep mode or $F_{A}=1$, the femtocell network would consume $17.6 \mathrm{~kW} / \mathrm{km}^{2}$ compared to $30.8 \mathrm{~kW} / \mathrm{km}^{2}$ for the macrocell network. As in this case, the femtocell network consumes $57 \%$ of the macrocell network, we see that the ability to put base stations in sleep mode is a key enabler to make a femtocell deployment a significant improvement compared to a macrocell deployment.

A high base station density leads on the one hand to more base stations and thus potential power consumers in the access network and on the other hand to a lower number of users per cell and associated lower $F_{A}$. When comparing two femtocell deployments, deployment $I$ is favorable over deployment $I I$ when:

$$
D_{B}^{I}\left[P_{s}^{F}+F_{A}^{I}\left(P_{\text {act }}^{F}-P_{s}^{F}\right)\right]<D_{B}^{I I}\left[P_{s}^{F}+F_{A}^{I I}\left(P_{\text {act }}^{F}-P_{s}^{F}\right)\right]
$$

When assuming $P_{s}^{F}$, the power consumption in sleep mode, is negligible, this simplifies to:

$$
D_{B}^{I} F_{A}^{I}<D_{B}^{I I} F_{A}^{I I}
$$

When assuming a hexagonal grid deployment:

$$
F_{A}^{I} / r^{I^{2}}<F_{A}^{I I} / r^{I I^{2}}
$$

or

$$
F_{A}^{I} / F_{A}^{I I}<r^{I^{2}} / r^{I I^{2}}
$$

When comparing deployments with channel bandwidths of $5 \mathrm{MHz}$ (I) and $20 \mathrm{MHz}$ (II), $r^{I^{2}} / r^{I^{2}}=36 \%$. The proportion of active base station fractions is 45 to $70 \%$. Thus, the $5 \mathrm{MHz}$ deployment is less power consuming, independent of $\alpha$.

\subsection{Life cycle assessment}

The use of power consumption as an environmental parameter is closely related to the requirement to reduce carbon emissions. However, when evaluating carbon emissions, the power consumption during the use of a device is only one part of the impact. When assessing carbon emissions, or other environmentally impacting factors, life cycle assessment (LCA) is often used. In LCA, the impacts during material extraction, manufacturing, transport, use and end-of-life are evaluated. In the case of carbon emissions, the use phase impact is directly correlated to the power consumption. By multiplying the average power consumption as given in (13) with the life time $l$ and the carbon emission intensity $I$, 
which denotes the amount of $\mathrm{CO}_{2}$ emitted when producing $1 \mathrm{kWh}$ of electrical power, one can calculate the use phase impact. Analysis of the other phases leads to an impact $L$, expressed in $\mathrm{kg} \mathrm{CO}_{2}$ per device. The latter impact cannot be influenced by $F_{A}$ and will be an important factor when evaluating the overall environmental desirability of deploying new mobile access networks.

\section{Use of the heuristic in a practical deployment}

As explained, when designing a mobile access network providing a large coverage for high bit rates, we need to deploy base stations with a high density. Using femtocells, we can create a more energy efficient access network. This was derived based on a theoretical situation with a hexagonal grid. Therefore, we would like to apply the derived heuristic in a more realistic situation.
We applied a genetic heuristic as used by mobile operators to deploy a set of LTE femtocells in an urban environment (Ghent, Belgium). The target for the heuristic was to provide a $95 \%$ coverage at a bit rate of 11.3 Mbps using a channel bandwidth of $5 \mathrm{MHz}$. On a surface of approximately $56000 \mathrm{~m}^{2}, 85$ femtocells were deployed resulting in a density of 1518 base stations/ $\mathrm{km}^{2}$. Compared to a theoretical density of 320 base stations $/ \mathrm{km}^{2}$ when using a hexagonal grid, we see the practical deployment needs about 4,7 times more base stations.

Using this environment, we randomly distributed users at a user density of 500 users $/ \mathrm{km}^{2}$ and $\alpha=1$. In this simulation we omitted the users that can not connect to any base station and replaced them by other users in order to sustain the required user density and bit rate requirement distribution. The simulation,

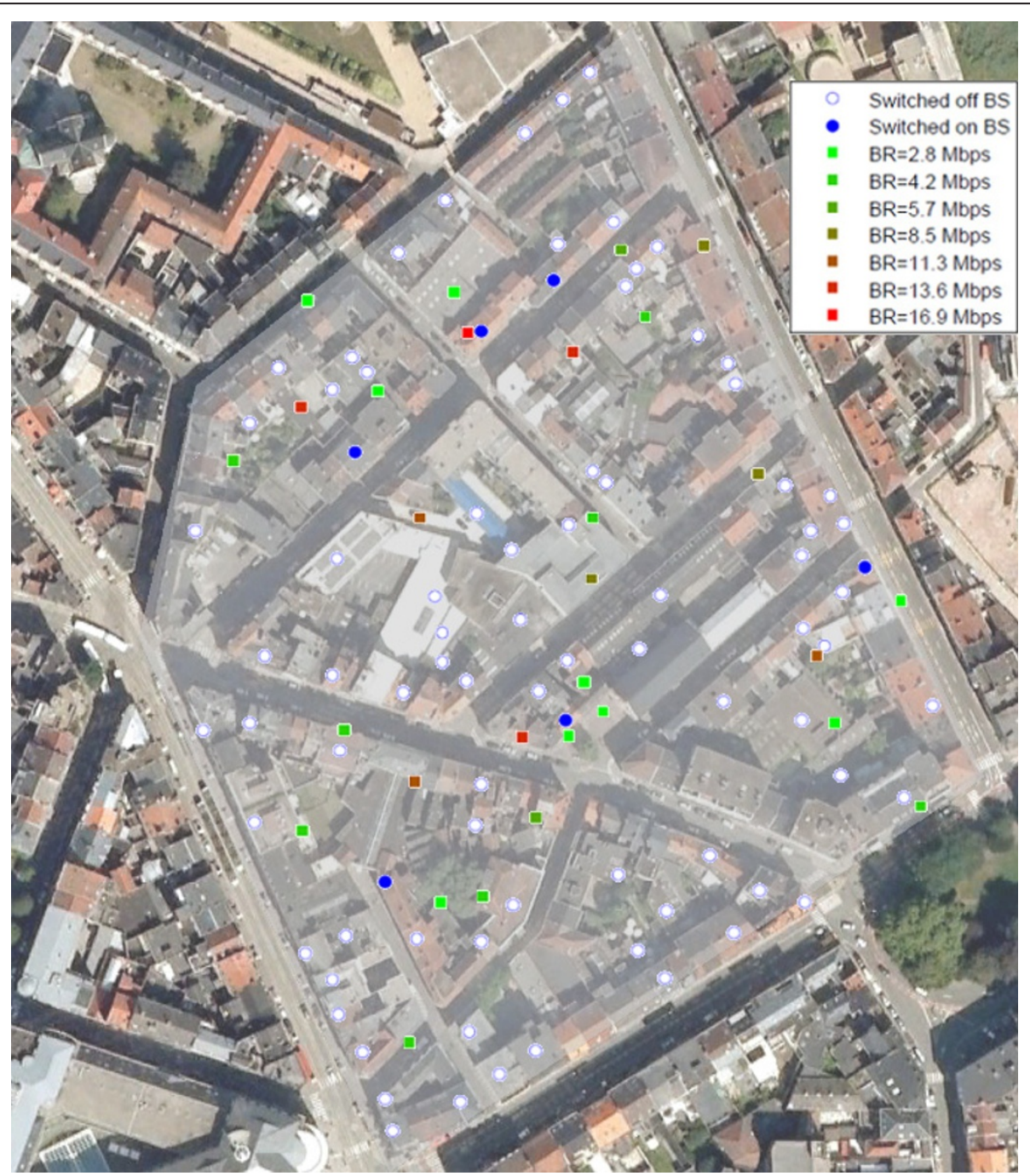

Figure 7 Sleep mode heuristic in a real life deployment $\left(D_{U}=500, \alpha=1\right)$. 
displayed in Figure 7, resulted in 6 base stations to be switched on or $F A=7.1 \%$. Assuming $P_{a c t}^{F}=10 \mathrm{~W}$ and $P_{s}^{F}=0 \mathrm{~W}$, this would result in an average power consumption of $60 \mathrm{~W}$ in this area. A simulation performed on a hexagonal grid designed to cover for a bit rate of $11.3 \mathrm{Mbps}$ with the same user behaviour resulted in an active base station fraction $F_{A}=28.7 \%$, or $51 \mathrm{~W}$ to cover the same surface. Comparing both power consumptions, we see that the heuristic limits the effect of the much higher base station density in the practical deployment. This implies that for the environmental impacts which are related to the number of active base stations, the results of an evaluation in a hexagonal grid will be a good prediction.

\section{Conclusions}

In light of the requirement for both increasing bit rates and reduced energy consumption in mobile access networks, the introduction of sleep modes is a key element. As it is difficult to analytically predict the energy saving potential that can be achieved by using sleep modes in mobile access network deployments, we have derived a heuristic that can serve as a design tool for establishing a baseline.

Due to the small cell size, femtocell access networks appear to be better suitable than macrocell networks for introducing sleep modes. At user densities between 100 and 1000 users the optimal active base station fraction for high bit rate networks is below $20 \%$. Thus, covering areas for high bit rates with sleep-enabled femtocells has a high potential for being less power consuming than using macrocells. However, also in macrocell networks there are opportunities for introducing sleep modes, especially when users have a preference for lower bit rates.

In practical deployments, where the distribution of base stations is suboptimal compared to a hexagonal grid deployment, the introduction of sleep modes helps in optimising the use of the base stations and reduces the effects of increased power consumption due to this suboptimal deployment.

\section{Acknowledgements}

The research leading to these results had received funding from the European Union Seventh Framework Programme (FP7/2007-2013) under grant agreement n. 257740 (Network of Excellence "TREND") and the IBBTproject Green ICT. W. Joseph was a Post-Doctoral Fellow of FWO-V (Research Foundation Flanders).

\section{Competing interests}

The authors declare that they have no competing interests.

Received: 30 August 2011 Accepted: 15 May 2012 Published: 15 May 2012

\section{References}

1. W Vereecken, W Van Heddeghem, M Deruyck, B Puype, B Lannoo, W Joseph, D Colle, L Martens, P Demeester, Power consumption in telecommunication networks: overview and reduction strategies. Communications Magazine, IEEE. 49(6), 62-69 (2011)

2. J Malmodin, A Moberg, D Lundn, G Finnveden, N Lövehagen, Greenhouse Gas Emissions and Operational Electricity Use in the ICT and Entertainment \& Media Sectors. Journal of Industrial Ecology. 14(5), 770-790 (2010). doi:10.1111/j.1530-9290.2010.00278x

3. M Pickavet, W Vereecken, S Demeyer, P Audenaert, B Vermeulen, C Develder, D Colle, B Dhoedt, P Demeester, Worldwide energy needs for ICT: The rise of power-aware networking, in Advanced Networks and Telecommunication Systems, 2008. ANTS '08. 2nd International Symposium on, [Bombay, India], 2, 1-3 (2008)

4. Cisco VNI Mobile, Global Mobile Data Traffic Forecast Update 2010 - 2015. Tech rep Cisco (2011)

5. 3GPP, LTE: 3rd Generation Partnership Project: Technical Specification Group Radio Access Network: Evolved Universal Terrestrial Radio Access (E-UTRA): User Equipment (UE) radio transmission and reception (TS 36.101 v 9.1.0 Release 9) (2009)

6. CE Jones, KM Sivalingam, P Agrawal, JC Chen, A Survey of Energy Efficient Network Protocols for Wireless Networks. Wireless Networks. 7, 343-358 (2001). doi:10.1023/A:1016627727877

7. G Fusco, H Gupta, $\epsilon$-Net Approach to Sensor k-Coverage. EURASIP Journal on Wireless Communications and Networking. 2010, 1-12 (2010). [Article ID 192752]

8. G Simon, M Molnar, L Gonczy, B Cousin, Robust k-Coverage Algorithms for Sensor Networks. Instrumentation and Measurement, IEEE Transactions on. 57(8), 1741-1748 (2008)

9. $Y$ Xiao, Energy saving mechanism in the IEEE 802.16e wireless MAN. Communications Letters, IEEE. 9(7), 595-597 (2005). doi:10.1109/ LCOMM.2005.1461675

10. J Zhou, M Jacobsson, I Niemegeers, Link Quality-Based Transmission Power Adaptation for Reduction of Energy Consumption and Interference. EURASIP Journal on Wireless Communications and Networking. 2010, 1-17 (2010). [Article ID 920131]

11. G Schmitt, The Green Base Station, in Telecommunication - Energy Special Conference (TELESCON), 2009 4th International Conference on, [Vienna, Austria], 4, 1-6 (2009)

12. D Valerdi, Q Zhu, K Exadaktylos, S Xia, M Arranz, R Liu, D Xu, Intelligent energy managed service for green base stations, in GLOBECOM Workshops (GC Wkshps), 2010 IEEE, [Miami, Florida], 29, 1453-1457 (2010)

13. V Mancuso, S Alouf, Reducing costs and pollution in cellular networks. Communications Magazine, IEEE. 49(8), 63-71 (2011)

14. L Correia, D Zeller, O Blume, D Ferling, Y Jading, I Godor, G Auer, L Van Der Perre, Challenges and enabling technologies for energy aware mobile radio networks. Communications Magazine, IEEE. 48(11), 66-72 (2010)

15. L Saker, SE Elayoubi, T Chahed, Minimizing Energy Consumption via Sleep Mode in Green Base Station, in Wireless Communications and Networking Conference (WCNC), 2010 IEEE, [Sydney, Australia], 12, 1-6 (2010)

16. I Ashraf, F Boccardi, L Ho, Sleep mode techniques for small cell deployments. Communications Magazine, IEEE. 49(8), 72-79 (2011)

17. M Marsan, L Chiaraviglio, D Ciullo, M Meo, Optimal Energy Savings in Cellular Access Networks, in Communications Workshops, 2009. ICC Workshops 2009. IEEE International Conference on, [Dresden, Germany], 44, 1-5 (2009)

18. E Oh, B Krishnamachari, Energy Savings through Dynamic Base Station Switching in Cellular Wireless Access Networks, in Global Telecommunications Conference (GLOBECOM 2010), 2010 IEEE, [Miami, Florida], 29, 1-5 (2010)

19. K Dufkova, M Bjelica, B Moon, L Kencl, JY Le Boudec, Energy savings for cellular network with evaluation of impact on data traffic performance, in Wireless Conference (EW), 2010 European, 916-923 (2010)

20. K Samdanis, D Kutscher, M Brunner, Self-organized energy efficient cellular networks, in Personal Indoor and Mobile Radio Communications (PIMRC), 2010 IEEE 21st International Symposium on, [Istanbul, Turkey], 21, 1665-1670 (2010)

21. Z Niu, Y Wu, J Gong, Z Yang, Cell zooming for cost-efficient green cellular networks. Communications Magazine, IEEE. 48(11), 74-79 (2010) 
22. M Deruyck, E Tanghe, W Joseph, L Martens, Modelling and optimization of power consumption in wireless access networks. Elsevier Computer Communications. 34(17), 2036-2046 (2010)

23. M Deruyck, D De Vulder, W Joseph, L Martens, Modelling the Power Consumption in Femtocell Networks, in IEEE Wireless Communications and Networking Conference (WCNC), [Paris, France], (2012)

24. V Erceg, LJ Greenstein, SY Tjandra, SR Parkoff, A Gupta, B Kulic, AA Julius, R Bianchi, An Empirically Based Path Loss Model for Wireless Channels in Suburban Environments. IEEE Journal on Selected Areas in Communications. 7(7), 1205-1211 (1999)

25. Recommendation ITU-R P1238-2, Propagation data and prediction methods for the planning of indoor radiocommunication systems and radio local area networks in the frequency range $900 \mathrm{MHz}$ to $100 \mathrm{GHz}$. (1997-2001)

26. IBM, IBM ILOG CPLEX Optimizer.http://www-01.ibm.com/software/ integration/optimization/cplex-optimizer/

doi:10.1186/1687-1499-2012-170

Cite this article as: Vereecken et al: Evaluation of the potential for energy saving in macrocell and femtocell networks using a heuristic introducing sleep modes in base stations. EURASIP Journal on Wireless Communications and Networking 2012 2012:170.

\section{Submit your manuscript to a SpringerOpen ${ }^{\mathcal{O}}$ journal and benefit from:}

- Convenient online submission

- Rigorous peer review

- Immediate publication on acceptance

- Open access: articles freely available online

- High visibility within the field

- Retaining the copyright to your article

Submit your next manuscript at $\gg$ springeropen.com 